\title{
Some Notes on Boltzmann and Landauer Phonon Thermal Transport at Nanoscale
} Amelia Carolina Sparavigna ${ }^{1}$

\author{
${ }^{1}$ Department of Applied Science and Technology, Politecnico di Torino, Italy
}

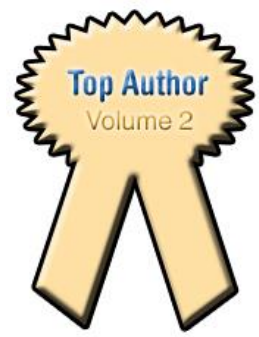

Abstract: To evaluate the phonon thermal transport at the nanoscale of nanotubes and nanowires we can use Boltzmann and Landauer approaches. The Boltzmann equation is coming from a semiclassical formulation, whereas the Landauer equation is based on ballistic models. Here we show, for teaching purposes, a simple manner of linking these two approaches using dimensional equations.

Keywords: Nanotubes, Nanowires, Nanostructures, Phonons, Thermal Transport, Relaxation Time Approximation, Boltzmann Equation, Landauer Formula.

\section{Introduction}

Nanostructures, for instance nanotubes, nanowires and nanoribbons, are structures with remarkable electronic and mechanical properties, which stimulate several theoretical and experimental studies. Electronic and lattice properties, such as specific heats and thermal transport coefficients, of nanotubes and nanowires had been the subject of a large number of researches and are continuing to attract new investigations [1-8]. Recently, other nanoscale objects, graphene and nanoribbons, had been added as subjects of investigation $[9,10]$.

The thermal transport in nanosystems is quite interesting [4]. At low temperatures, the behaviour of thermal conductance in carbon nanotube bundles follows the power law $T^{\alpha}$, where $T$ is the temperature and the exponent $\alpha$ is about 1.5. This shows that thermal transport in the bundle has an intermediate behaviour between that of onedimensional systems and that of two-dimensional structures. For nanowires, according to the wire section, $\alpha$ is ranging from 1 to 3 . This behaviour of the thermal conductivity comes from sets of lattice vibrations where quantization is important.

Here we will discuss Boltzmann and Landauer methods to evaluate thermal transport, as proposed in $[11,12]$, for nanotubes and nanowires. This discussion could be useful for teaching purposes, because it is showing a simple manner of linking these two approaches, one based on a semiclassical theory, the other on ballistic models.

\section{Phonons and thermal transport}

In a dielectric material, where freely moving charges are not present, the thermal transport is given by phonons. These quasi-particles are coming from the quantization of the elastic vibrations of lattice. When the material vibrates, we have a displacement field of lattice positions, $\boldsymbol{\eta}_{b}(\mathbf{h})$, where $\mathbf{h}$ is the position vector of the unit cell composed of $b$ masses.

The displacement fields of atoms on nanotubes can be composed in the similar manner, as usually done for three- dimensional systems [14-18], with the following functions:

$\mathbf{u}_{b}(\mathbf{h})=\boldsymbol{\varepsilon}_{q, p}^{b} e^{i \mathbf{q} \cdot \mathbf{h}}$

where $\mathbf{q}$ is the wave-vector and $\boldsymbol{\varepsilon}_{q}^{b}$ the polarization vector. The expansion of displacement field $\boldsymbol{\eta}_{b}(\mathbf{h})$ is then:

$\boldsymbol{\eta}_{b}(\mathbf{h})=i \sqrt{\frac{\hbar}{M_{b} N}} \cdot$
$\sum_{q, p} \frac{1}{\sqrt{\omega_{q, p}}}\left[\mathbf{u}_{b}^{*}(\mathbf{h}) a_{q, p}-\mathbf{u}_{b}(\mathbf{h}) a_{q, p}^{+}\right]$

$N$ is the number of the lattice points, $M_{b}$ the atomic mass at position $b$ in the cell, and $\omega_{q, p}$ the angular frequency for a given wave vector $\mathbf{q}$ and polarization p. As in [19], we can calculate the phonon dispersions.

In $[11,12]$, we obtained the angular frequencies of phonons, traveling along the axis of the nanotube (see Figure 1). Like the continuous models for hollow tubes, four acoustic modes are found. Two of them are the twisting mode in tangential direction and the longitudinal stretching mode in the direction of the tube. The other two acoustic modes are flexural 
oscillations, where the center-of-mass of each tube section is moving perpendicularly with respect to the tube axis. This flexural motion has a double degeneracy in the $\mathbf{x , y}$ plane perpendicular to $\mathbf{z}$. Breathing modes in the radial direction are also present, but they have a finite frequency at $q_{z}=0$, due to the curvature of the lattice.
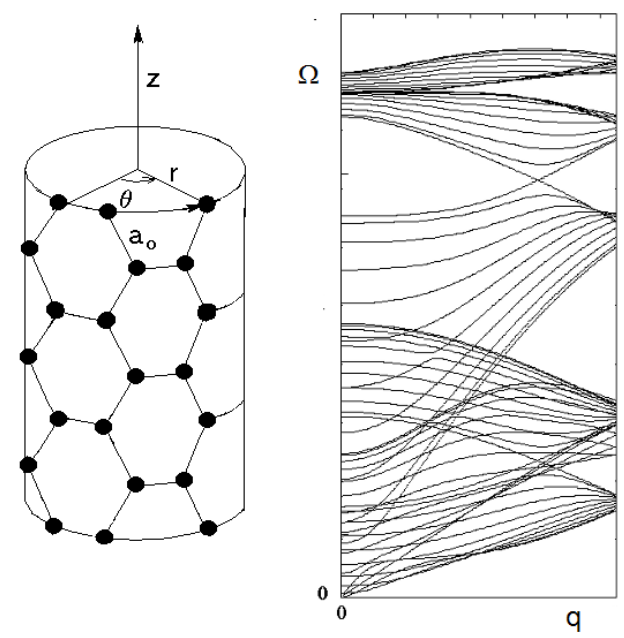

Figure 1: Armchair lattice, with lattice constant $a_{o}$, rolled up on a tube in achiral configuration. On the right, we have the angular frequency as a function of wavenumber $q_{z}$ for a $(10,10)$ tube. One of the acoustic branches is two-fold degenerate.

\section{The Boltzmann approach}

Let us discuss the thermal transport in the framework of the time relaxation approximation. The phonon thermal conductivity in lattices is linked to the perturbed phonon distribution $n_{Q}$. From now on, $Q$ is the abbreviate notation for $\vec{q}, p$.

We can define a deviation function $\psi_{Q}$ as:

$n_{Q}-n_{Q}^{o}=-\psi_{Q} \frac{\partial n_{Q}^{o}}{\partial\left(\hbar \omega_{Q}\right)}$

where $n_{Q}^{o}$ is the unperturbed phonon distribution. A linearized Boltzmann equation, for a solid subjected to a thermal gradient, can be written in the relaxation time approximation as [13]:

$$
\begin{aligned}
k_{B} T \mathbf{v}_{Q} \cdot \vec{\nabla} T \frac{\partial n_{Q}^{o}}{\partial T} \\
\quad=-\frac{1}{\tau_{Q}} \psi n_{Q}^{o}\left(1+n_{Q}^{o}\right)
\end{aligned}
$$

where $\mathbf{v}_{Q}=\partial \omega_{Q} / \partial \mathbf{q}$ is the phonon group velocity and $\tau_{Q}$ the phonon relaxation time. $k_{B}$ is the Boltzmann constant and $\vec{\nabla} T$ the gradient of temperature.

The heat current density is defined, for a nanotube with volume $V=S L$, where $S$ is the section and $L$ the length, as:

$$
\begin{aligned}
& \vec{U}=\frac{1}{V} \sum_{Q} \hbar \omega_{Q} \mathbf{v}_{Q} n_{Q}= \\
& =-\frac{1}{2 \pi S} \sum_{\mu, p} \int d q_{z} \hbar \omega_{Q} v_{Q} \psi_{Q} \frac{\partial n_{Q}^{o}}{\partial\left(\hbar \omega_{Q}\right)}
\end{aligned}
$$

Here, we imagined an infinitely long tube and then substituted the sum on the lattice wavenumbers along the axis of the tube, the $\mathrm{z}$-axis, with an integral. $\mu$ is an integer ranging from $-m / 2$ to $m / 2-1$, in an armchair $(m, m)$ tube, having $m$ atoms on its circumference [19].

The thermal conductivity is defined as the coefficient $\kappa$ joining the heat current with the thermal gradient as $\vec{U}=\kappa \vec{\nabla} T$. We can use a reduced frequency and a dimensionless variable $\checkmark$ defined in the following manner:

$\omega_{Q}=a_{o} \sqrt{\frac{\Phi_{o}}{M}} \bar{\omega}_{Q} ; \varsigma=\frac{a_{o}}{2 \pi} q_{z}$

$a_{o}$ is the lattice constant. In (6), we have a scale factor $\Phi_{o}$ for the potential. Its dimensions are mass $\cdot$ length $^{-2} \cdot$ time $^{-2}$. Let us guess the phonon relaxation time as given by $L / \tau_{Q}=\partial \omega_{Q} / \partial \mathbf{q}$ [11]; the thermal conductivity turns out to be, after simple calculations:

$$
\begin{gathered}
\kappa=\frac{\hbar^{2} a_{o}^{3} L}{k_{B} T^{2} S}\left(\frac{\Phi}{M}\right)^{\frac{3}{2}} . \\
\cdot \sum_{\mu, p} \int d \varsigma \bar{\omega}_{Q}{ }^{2} \frac{\partial \bar{\omega}_{Q}}{\partial \varsigma} n_{Q}^{o}\left(1+n_{Q}^{o}\right)
\end{gathered}
$$

The comparison with experimental data is given in Ref.11 and also in [20]. Let us remark just the following fact: $L$, the length of the nanotube, is also 
the distance between the heater resistor and the sensor resistor in experimental set-ups.

\section{The Landauer approach}

The Landauer approach was developed for the physics of electronic transport in one dimension. This physics is quite important due to its remarkable observed phenomena, like quantum Hall effect and quantized conductance of ballistic point contacts. The presence of quantization in these phenomena is displayed by a conductance, which is multiple of the fundamental quantum $e^{2} / h$. The frameworks of Landauer and Büttiker-Landauer theories were used to explain quantization [21-23].

One-dimensional phonon transport should also be possible, in nanowires and nanotubes. In Ref.23, authors show that, in a low temperature regime dominated by ballistic massless phonon modes, the phonon thermal conductance of a one-dimensional quantum wire is quantized, the fundamental quantum of thermal conductance $G$ being:

$$
G=\frac{\pi^{2} k_{B}^{2} T}{3 h}
$$

where $h$ is Planck's constant.

It is possible to link the Boltzmann expression of the energy flux to the Landauer expression of ballistic transport [11,23]:

$$
\begin{aligned}
& \dot{Q}=\vec{U} \cdot \vec{S}= \\
& =\sum_{\alpha} \int \frac{d q}{2 \pi} \hbar \omega_{\alpha} v_{\alpha}\left(n_{R}-n_{L}\right) P_{\alpha}
\end{aligned}
$$

carried out in quantum nanowires or nanotubes from left $\mathrm{L}$ to right $\mathrm{R}$ phonon reservoirs, both described by the equilibrium phonon distribution. $\alpha$ is counting polarization and phonon bands. $P_{\alpha}$ is the transmission coefficient of the wire.

Let us write Eq.5 as:

$$
\begin{aligned}
& \dot{Q}=-\frac{1}{2 \pi} \sum_{\alpha} \int d q \hbar \omega_{Q} v_{Q} \psi_{Q} \frac{\partial n_{Q}^{o}}{\partial\left(\hbar \omega_{Q}\right)}= \\
& =-\frac{1}{2 \pi k_{B} T} \sum_{\alpha} \int d q \hbar \omega_{Q}^{2} v_{Q} \psi_{Q} n_{Q}^{o}\left(n_{Q}^{o}+1\right)
\end{aligned}
$$

Substituting $\quad \psi_{Q} \quad$ (see $\quad$ Eq.4) and
$L / \tau_{Q}=\partial \omega_{Q} / \partial q$, we have:

$$
\dot{Q}=-\frac{1}{2 \pi} \sum_{\alpha} \int d q \hbar \omega_{Q} v_{Q} \frac{\partial T}{\partial z} \frac{\partial n_{Q}^{o}}{\partial T} L
$$

This expression can be further reduced to:

$$
\dot{Q}=-\frac{1}{2 \pi} \sum_{\alpha} \int d q \hbar \omega_{Q} v_{Q} \frac{\Delta n}{\Delta z} L
$$

In this last equation, where we are passing from a diffusive description to a ballistic one, we are thinking of a phonon population which is coming from the reservoirs; however, let us note that these phonons have the dispersions proper of the tube or wire connecting the thermal baths [11]. If the distance between the reservoirs is equal to the tube length $L$ :

$$
\dot{Q}=-\frac{1}{2 \pi} \sum_{\alpha} \int d q \hbar \omega_{Q} v_{Q}\left(n_{L}-n_{R}\right)
$$

This is the Landauer formula for transmission coefficients equal to 1 .

This approach, proposed in [11], shows a simply manner of linking the semiclassical Boltzmann equation to the expression of Landauer ballistic transport. It is also possible of obtaining a thermal conductance, as in Ref.23.

According to a diffusive model, the conductance $G$ of a nanotube is:

$$
\begin{gathered}
G=\frac{\hbar^{2} a_{o}^{3}}{k_{B} T^{2}}\left(\frac{\Phi_{o}}{M}\right)^{\frac{3}{2}} . \\
\sum_{\mu, p} \int \varsigma \bar{\omega}_{Q}^{2} \frac{\partial \bar{\omega}_{Q}}{\partial \varsigma} n_{Q}^{o}\left(1+n_{Q}^{o}\right)
\end{gathered}
$$

Of course, this expression is different from the Landauer conductance, which is:

$G=g \frac{\pi^{2} k_{B}^{2}}{3 h} T=\frac{g \pi}{6} \frac{k_{B}^{2} T}{\hbar}=\frac{g \pi}{6} F$

In (15), $g$ is the number of acoustic modes; this formula works for temperatures lower than $2 \mathrm{~K}$. Let us stress the presence, in this conductance, of the dimensioned factor $F=k_{B}^{2} T / \hbar$.

To compare (14) and (15), we can use a dimensional approach. From (14), let us consider just the dimensioned factor: 


$$
\frac{\hbar^{2} a_{o}^{3}}{k_{B} T^{2}}\left(\frac{\Phi_{o}}{M}\right)^{3 / 2}
$$

From (6), we can define an angular frequency $\widetilde{\omega}$, as:

$$
\tilde{\omega}=a_{o} \sqrt{\frac{\Phi_{o}}{M}}
$$

Using this angular frequency in (16), we obtain:

$$
\begin{aligned}
& \frac{\hbar^{2} a_{o}^{3}}{k_{B} T^{2}}\left(\frac{\Phi_{o}}{M}\right)^{\frac{3}{2}}=\frac{\hbar^{2} \tilde{\omega}^{3}}{k_{B} T^{2}}= \\
& =\frac{\hbar^{2}}{k_{B} T^{2}}\left(\frac{\hbar \tilde{\omega}}{k_{B} T}\right)^{3}\left(\frac{k_{B} T}{\hbar}\right)^{3}
\end{aligned}
$$

After simplifying we have:

$$
\begin{aligned}
& \frac{\hbar^{2} a_{o}^{3}}{k_{B} T^{2}}\left(\frac{\Phi_{o}}{M}\right)^{\frac{3}{2}}=\frac{k_{B}^{2} T}{\hbar}\left(\frac{\hbar \tilde{\omega}}{k_{B} T}\right)^{3}= \\
& =\frac{k_{B}^{2} T}{\hbar} \xi^{3}=F \xi^{3}
\end{aligned}
$$

Being $\xi$ dimensionless, we have that the dimensioned factor $F$ of the Landauer formula is the same of that of the conductance found by means of the Boltzmann approach.

For what concerns the Landauer expression (15), let us remember that in carbon nanotubes and in nanowires there are four acoustic modes: one longitudinal, one torsional, and two flexural modes. And then, the low temperature thermal conductivity have four quanta of thermal conductance.

\section{References}

1. Yi, W., Lu, L., Dian-lin, Z., Pan, Z.W., and Xie, S.S. (1999). Linear specific heat of carbon nanotubes, Phys. Rev. B 59, R9015-R9018.

2. Hone, J., Whitney, M., Piskoti, C., and Zettl, A. (1999). Thermal conductivity of single-walled carbon nanotubes, Phys. Rev. B 59, R2514-R2516.

3. Kim, P., Shi, L., Majumdar, A., and McEuen, P.L. (2001). Thermal transport measurements of individual multiwalled nanotubes, Phys. Rev. Lett. 87, 215502 (5 pages).

4. Li, D., Wu, Y., Kim, P., Shi, L., Yang, P., and Majudar, A. (2003). Thermal conductivity of individual silicon nanowires, Appl. Phys. Lett. 83, 2934-2936.

5. Dresselhaus, M.S., Dresselhaus, G., and Eklund, P.C. (1996). Science of fullerenes and carbon nanotubes, Academic Press, New York.

6. Behabtu, N., Young, C.C., Tsentalovich, D.E., Kleinerman, O., Xuan Wang, X., Ma, A.W.K., Amram Bengio, E., ter Waarbeek, R.F., de Jong, J.J., Hoogerwerf, R.F., Fairchild, S.B., Ferguson, J.B., Maruyama, B., Kono, J., Talmon, Y., Cohen, Y., Otto, M.J., and Pasquali, M. (2013). Strong, light, multifunctional fibers of carbon nanotubes with ultrahigh conductivity, Science 339 (6116), 182-186.
7. Salaway, R.N., and Zhigilei, L.V. (2014). Molecular dynamics simulations of thermal conductivity of carbon nanotubes: Resolving the effects of computational parameters, International Journal of Heat and Mass Transfer 70, 954-964.

8. De Volder, M. F., Tawfick, S. H., Baughman, R. H., and Hart, A. J. (2013). Carbon nanotubes: present and future commercial applications, Science 339 (6119), 535-539.

9. Ouyang, T., Chen, Y., Liu, L.-M., Xie, Y., Wei, X., and Zhong, J. (2012). Thermal transport in graphyne nanoribbons, Phys. Rev. B 85, 235436 (7 pages).

10. Zhong, W.-R., Zheng, D.-Q., and Hu, B. (2012). Thermal control in graphene nanoribbons: thermal valve, thermal switch and thermal amplifier, Nanoscale 4, 5217-5220.

11. Sparavigna, A., and Ravetti A. (2005). Thermal conductivity in nanotubes and nanotube bundles, Recent Res. Devel. Physics 6, 173-184, S.G. Pandalai Ed., ISBN 81-7895-171-1.

12. Sparavigna, A. (2008). Lattice specific heat of carbon nanotubes, Journal of Thermal Analysis and Calorimetry 93, 983-986.

13. Ziman, J.M. (1960). Electrons and phonons: the theory of transport phenomena in solids, Clarendon, Oxford.

14. Srivastava, G.P. (1990). The physics of phonons, Hilger, Bristol.

15. Sparavigna, A. (2002). Influence of isotope scattering on the thermal conductivity of diamond, Phys. Rev. B 65, 064305 (5 pages)

16. Omini, M., and Sparavigna, A. (1997). Heat transport in dielectric solids with diamond structure, Nuovo Cim. D 19, 1537-1563.

17. Omini, M., and Sparavigna, A. (1997). Beyond the isotropicmodel approximation in the theory of thermal conductivity, Phys. Rev. B 53, 9064-9073.

18. Sparavigna, A.C., and Galli, S. (2012). L'equazione di Boltzmann per la conducibilità termica fononica nell'approssimazione dei tempi di rilassamento, Lulu Enterprises, Raleigh, NC.

19. Mahan, G.D., and Gun Sang Jeon, (2004). Flexure modes in carbon nanotubes, Phys. Rev. B, 70, 075405 (11pages).

20. Sparavigna, A. (2006). Lattice specific heat of carbon nanotubes, arXiv:cond-mat/0609139 [cond-mat.mtrl-sci].

21. Imry, J. (1997). Introduction to Mesoscopic Physics, Oxford University Press.

22. Datta, A. (1995). Electronic transport in mesoscopic systems, Cambridge University Press.

23. Rego, L.G.C., and Kirczenow, G. (1998). Quantized thermal conductance of dielectric quantum wires, Phys. Rev. Lett. 81, 232-235. 